\title{
Effets sur le spermatozoïde humain du Diuron (3-(3,4-dichlorophényl)-1,1-diméthyl-urée) et de l'un de ses produits de transformation, la 3,4- dichloroaniline (3,4-DCA) (Etude préliminaire)
}

\author{
C. MALPUECH-BRUGERE1, G. GRIZARD1, P. BOULE 2 , D. BOUCHER1 \\ 1 Laboratoire de Biologie de la Reproduction, Faculté de médecine, UFR d'Auvergne - Clermont I, \\ CECOS Hôtel Dieu, Clermont-Ferrand 63000. \\ 2 Laboratoire de Photochimie Moléculaire et Macromoléculaire - UFR Blaise Pascal - Clermont II, \\ Aubière 63120.
}

\section{RÉSUMÉ}

Le Diuron est un herbicide appartenant à la famille des halogénophénylurée. Un test de toxicité basé sur l'inhibition de la bioluminescence d'une bactérie marine Vibrio fischeri, comme indicateur de toxicité a montré que les produits de bio- ou photo-transformation sont plus toxiques que le Diuron lui-même. Tous ces composés sont des molécules très lipophiles et une membrane cellulaire riche en lipides comme celle du spermatozoïde pourrait être une cible privilégiée pour leur interaction avec la cellule. Des études sont en cours dans notre laboratoire afin d'évaluer les effets in vitro du Diuron et de l'un de ses produits de transformation, la 3,4-dichloroaniline (3,4-DCA) (obtenu par élimination de la fonction urée) sur les propriétés structurales et fonctionnelles du spermatozoïde humain. Les études sont réalisées sur des spermatozoïdes provenant de spermes normaux (critères de l'OMS), purifiés sur gradient discontinu de Percoll. Trois millions de spermatozoïdes/ml sont incubés dans un milieu synthétique de Earle dépourvu de rouge de phénol, supplémenté par $7,5 \%$ de sérum humain décomplémenté pendant 24 heures, à température ambiante, en présence de Diuron ou de 3,4-DCA $(0,1 ; 1 ; 5 \mathrm{mM})$ dans un volume final de $250 \mu$ l. Nos premiers résultats mettent en évidence une diminution de la vitalité et de la mobilité des spermatozoïdes après 24 heures d'incubation en présence de $5 \mathrm{mM}$ de Diuron. L'anisotropie (inversement proportionnelle à la fluidité membranaire) mesurée par polarisation de fluorescence en utilisant une sonde lipophile, le 1,6-Diphényl-1,3,5-Hexatriène est aussi diminuée. La 3,4-DCA entraîne des effets plus rapides et plus marqués que le Diuron. Tous les spermatozoïdes sont "morts" après 30 minutes ou 24 heures d'incubation, en présence respectivement de $5 \mathrm{mM}$ et $1 \mathrm{mM}$ de 3,4-DCA. Aux concentrations utilisées, ces molécules s'avèrent plus toxiques pour le spermatozoïde et cet effet semblerait être lié à une atteinte membranaire. Cette étude préliminaire suggère que des tests puissent être développés à partir des spermatozoïdes humains pour évaluer la toxicité de certains pesticides.

Mots clés : spermatozoïde, pesticide, membrane, fluidité

Correspondance : C. Malpuech-Brugere, Laboratoire de Biologie de la Reproduction, Faculté de médecine, UFR d'Auvergne - Clermont I, CECOS Hôtel Dieu, 63000 Clermont-Ferrand.

Communication orale sélectionnée au XVIIème Congrès de la SALF, 7-8 décembre 2000, Bordeaux. 


\section{INTRODUCTION}

Le spermatozoïde est une cellule hautement différenciée. Sa membrane plasmique est particulièrement riche en cholestérol et en phospholipides sur lesquels sont le plus souvent fixés des acides gras insaturés par exemple, l'acide docosahexanoïque (C22:6 n-3) [10, 12]. Cette composition particulière rend la membrane du spermatozoïde sensible au stress oxydant et à une peroxydation lipidique importante. Celle-ci entraîne notamment une désorganisation moléculaire de sa structure membranaire [3] révélée en particulier par des altérations de fluidité $[9,14]$ et par des altérations fonctionnelles portant en particulier sur la mobilité, la capacitation et la réaction acrosomique [8]. Compte tenu de cette structure membranaire particulière et du fait qu'il s'agit d'une cellule facilement accessible, le spermatozoïde humain pourrait être utilisé comme modèle pour étudier in vitro l'effet de certains pesticides de nature lipophile.

Parmi les pesticides, nous avons retenu le Diuron qui est un herbicide systémique, très peu soluble dans l'eau, appartenant à la famille des halogénophénylurées de type phénylurée (Figure 1) [17]. Il n'est pas très toxique, mais sa biotransformation ou sa phototransformation peut conduire à des composés beaucoup plus dangereux [17]. La bio-transformation passe en effet par une déméthylation de la fonction urée conduisant à la formation d'halogénoaniline (dichloroaniline) (Figure 1) [16]. La photolyse en absence d'eau, à l'état dispersé sur silice par exemple, entraîne également une déméthylation mais aussi une oxydation en dérivé formylé qui présente une $\mathrm{CE}_{50}$ (concentration nécessaire pour réduire de 50\% la luminescence de la bactérie marine Vibrio fischeri) beaucoup plus faible que celle du com-

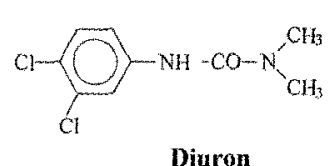

Diuron

Figure 1 : Représentation chimique des molécules utilisées dans l'étude. posé de départ $(7,3 \mathrm{mg} / \mathrm{L}$ respectivement pour les produits formylés, alors qu'elle est supérieure à $40 \mathrm{mg} / \mathrm{L}$ pour le Diuron).

Le but de cette étude est d'évaluer l'effet du Diuron et de la 3,4-DCA, à différentes concentrations, sur la vitalité et la mobilité des spermatozoïdes, deux critères essentiels pour apprécier la toxicité des molécules testées et sur la fluidité membranaire, un paramètre pour préciser éventuellement le niveau d'action de celles-ci.

\section{MATERIELS ET METHODES}

\section{Préparation des échantillons}

Les études sont réalisées à partir de spermes présentant des caractéristiques normales d'après les critères de l'OMS. Afin d'obtenir une suspension de spermatozoïdes, la plus homogène possible, les spermatozoïdes sont purifiés sur gradient discontinu de Percoll (47,5\% / 95\%) (Sigma Aldrich, St Quentin Fallavier, France). Le culot est lavé puis remis en suspension dans un milieu synthétique de Earle modifié (EBSS) (Earle balanced Salt Solution, Sigma) dépourvu de rouge de phénol, supplémenté par 7,5\% de sérum humain décomplémenté.

Le Diuron (Rhône-Poulenc, Lyon, France) et la 3,4 Dichloroaniline (3,4-DCA) (Sigma) sont mis en solution dans du diméthyl sulfoxyde (DMSO) (Sigma). Les spermatozoïdes (3 x 106) sont incubés dans des microplaques de 96 puits, pendant 24 heures, à température ambiante, en présence de Diuron ou de 3,4DCA $(0,1 ; 1 ; 5 \mathrm{mM})$ dans un volume final de $250 \mu$ l. Des témoins sont réalisés dans des conditions identiques avec le DMSO seul.

La vitalité est évaluée par l'exclusion de l'éosine (colorant vital), la nigrosine étant utilisée comme contre-colorant. La mobilité totale est appréciée de façon qualitative, elle peut être différenciée en mobilité progressive correspondant aux spermatozoïdes qui se déplacent suivant une trajectoire sensiblement linéaire et en mobilité non progressive, correspondant aux spermatozoïdes mobiles sur place ou ne progressant que très faiblement. Vitalité et mobilité sont appréciées en microscopie optique sur 100 spermatozoïdes. 
La fluidité membranaire est appréciée par mesure de l'anisotropie par polarisation de fluorescence en utilisant une sonde lipophile, le 1,6-Diphényl-1,3,5-Hexatriène (DPH) qui s'incorpore de façon homogène aux phospholipides de la membrane plasmique. Brièvement, après lavage dans du tampon phosphate (PBS) (Sigma) et centrifugation (1800 g, $10 \mathrm{~min}$ ), les spermatozoïdes sont remis en suspension dans $3 \mathrm{ml}$ de PBS contenant 10-6 M de DPH. La suspension cellulaire est alors incubée pendant 30 min à température ambiante et la lecture est réalisée à $37^{\circ} \mathrm{C}$ au moyen d'un spectrofluorimètre. Ce paramètre expérimental est inversement proportionnel à la fluidité membranaire [9].

\section{Expression des résultats}

Pour la mobilité et la vitalité, les résultats sont exprimés en pourcentage de variation de l'échantillon à tester par rapport au témoin correspondant. Les résultats rapportés sur les figures sont ceux d'un essai représentatif de trois expérimentations indépendantes.

\section{RESULTATS}

Après 24 heures d'incubation en présence de 5 $\mathrm{mM}$ de Diuron, il existe une diminution de la vitalité et de la mobilité. Pour les concentrations de Diuron 1 et $0,1 \mathrm{mM}$, ces deux paramètres sont peu modifiés (Figure 2). Après 24 heures d'incubation, l'anisotropie est diminuée pour les deux concentrations les plus élevées de Diuron ; ce qui traduit une augmentation de la fluidité (Tableau 1). Dans nos conditions expérimentales, l'effet de la 3,4-DCA est largement dépendant de la concentration utilisée ; en présence de $5 \mathrm{mM}$, la vitalité est très rapidement diminuée puisque après 30 minutes d'incubation, tous les spermatozoïdes sont

Tableau 1 : Effet du Diuron sur l'anisotropie (r) du DPH (moyenne \pm ESM (erreur standard moyenne), $n=3$ )
Concentration
Diuron (mM)
r DPH
0
$0,208 \pm 0,02$
$\mathbf{0 , 1}$
$0,213 \pm 0,02$
1,0
$0,191 \pm 0,003$
$\mathbf{5 , 0}$
$0,188 \pm 0,001$

"morts" (Figure 3). Pour les deux autres concentrations, une diminution de la vitalité et de la mobilité est observée à 24 heures (Figure 4). Compte tenu de la perte de vitalité après 24 heures d'incubation, les mesures de fluidité (anisotropie $=r$ ) ont seulement été évaluées pour la plus faible dose et pour le témoin sans pesticide (respectivement $\mathrm{r}=0,184$ et $\mathrm{r}=$ $0,189)$.

\section{DISCUSSION}

La plupart des études de toxicité des herbicides de type halogénophényl urée a été effectuée in vivo, sur des mammifères, notamment la souris et elles consistent à évaluer la toxicité en aiguë ou chronique des produits. Ainsi, le Diuron a des effets sur la moelle osseuse [1] ou encore sur la rate [18]. Des effets de la 3,4DCA sur l'hémoglobine ou encore sur l'immunité cellulaire ont aussi été décrits [6]. D'après les quelques travaux réalisés chez l'homme, il apparaît que le Diuron est faiblement toxique. Une étude récente réalisée sur des leucocytes humains a toutefois montré que le Diuron à des concentrations micromolaires entraîne une diminution de la production de certaines cytokines (IL-5, TNF $\alpha$ et IFN $\gamma$ ), suggérant ainsi que cet herbicide pourrait provoquer à faibles doses, une perturbation du système immunitaire [11].

Dans notre modèle expérimental, la 3,4-DCA s'avère plus toxique que le Diuron. Ceci confirme des résultats précédants utilisant le test de toxicité de Vibrio fischeri [16]. Par ailleurs, nos résultats sont en accord avec des travaux récents [7] montrant que d'autres pesticides (le parathion et le paraoxon) provoquent des altérations membranaires de façon dose-dépendante et une diminution de la vitalité des spermatozoïdes. Classiquement, il est admis qu'une perte de la vitalité entraîne une rigidification donc une augmentation de l'anisotropie [9]. Cette observation est apparemment contradictoire avec les résultats de cette étude. En fait, l'augmentation de la fluidité observée après incubation des spermatozoïdes en présence des pesticides, reflète probablement des modifications ultra-structurales de la membrane. En s'intercalant dans la bicouche phospholipidique, la sonde fluorescente (le DPH) se 


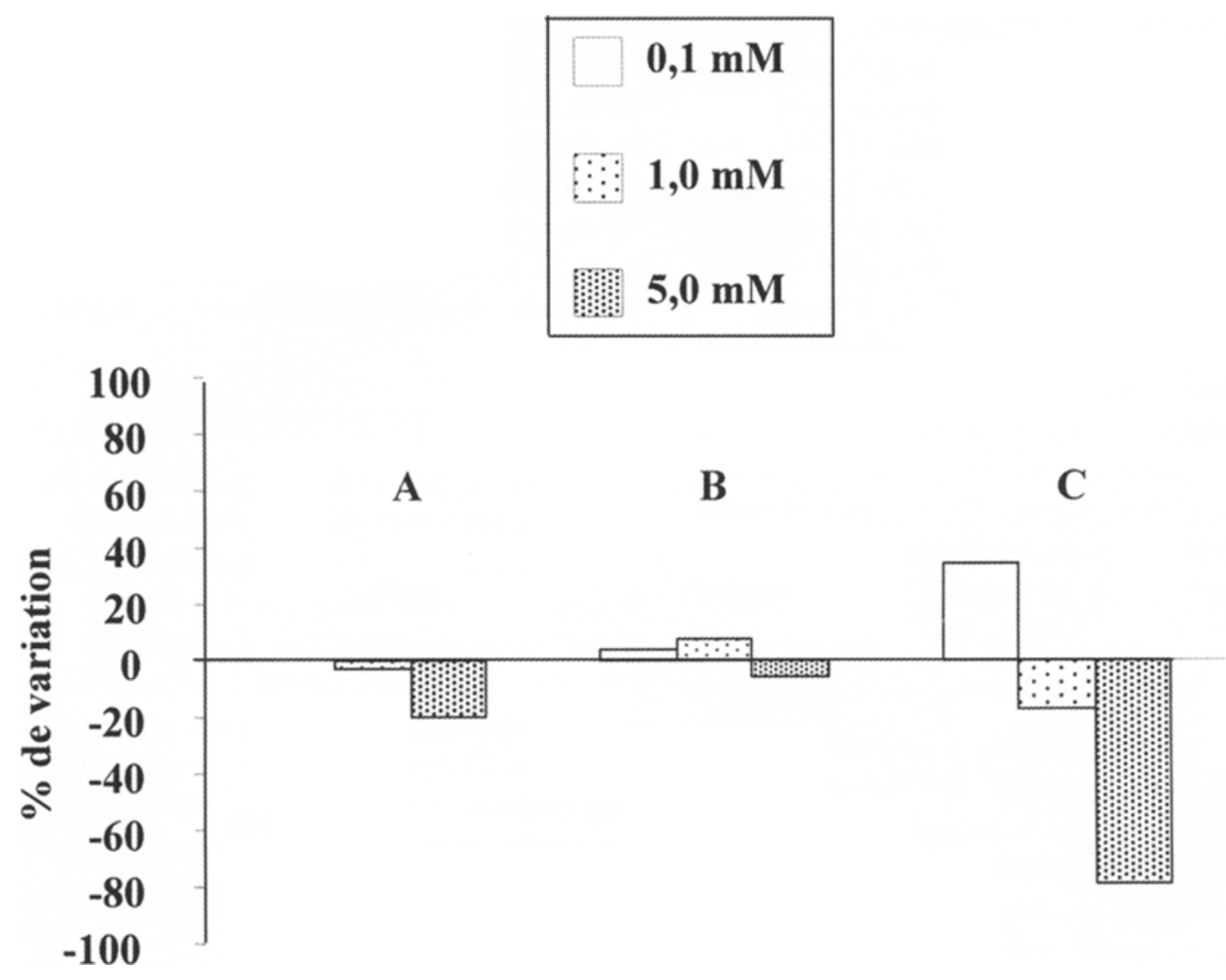

Figure 2 : Effet du Diuron sur la vitalité (A) et la mobilité totale (B) et progressive (C).

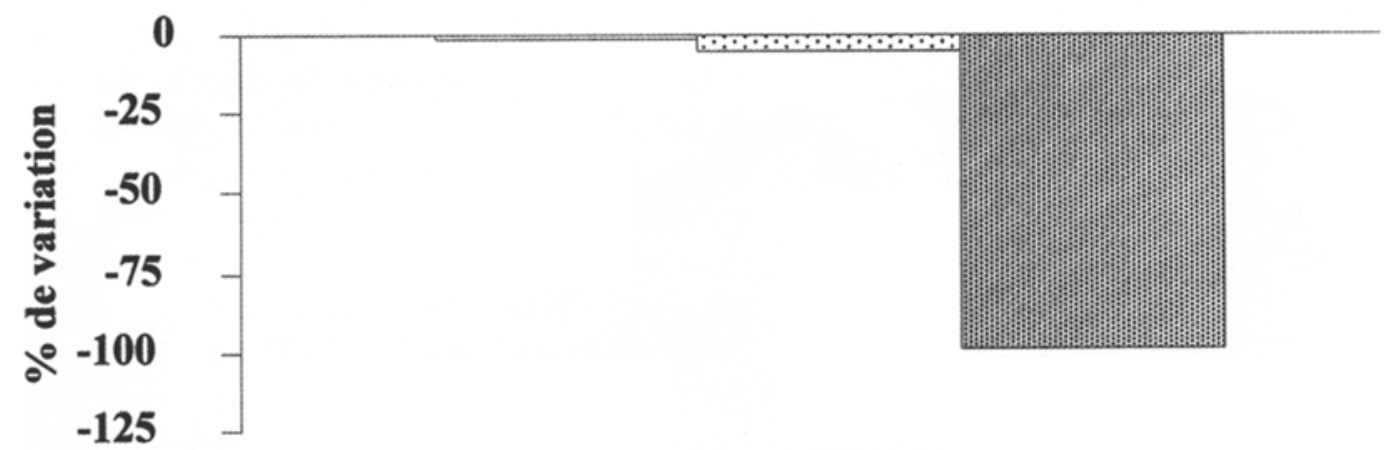

Figure 3 : Effet de la 3,4 DCA sur la vitalité après 30 minutes d'incubation. 


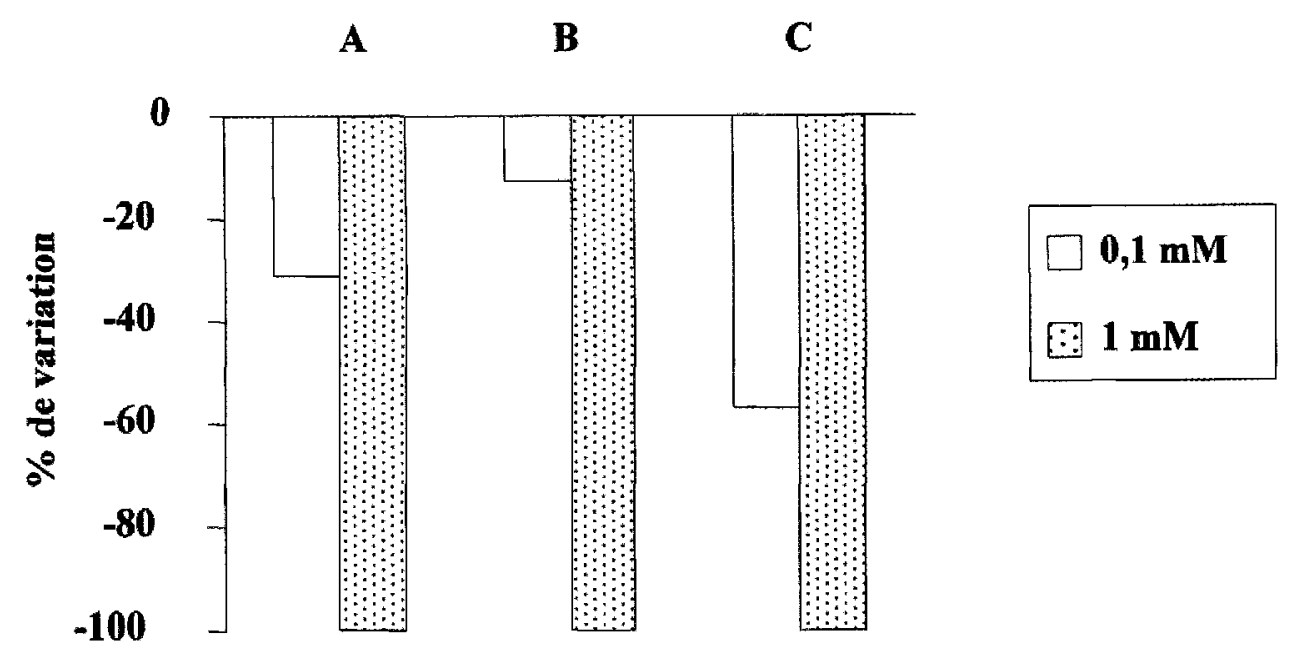

Figure 4 : Effet de la 3,4-DCA sur la vitalité (A )et la mobilité totale (B) et progressive (C) après 24 heures d'incubation.

comporte comme un phospholipide et les différences d'anisotropie observées reflètent un changement dans l'environnement immédiat de la sonde. Elles traduisent des modifications de la dynamique lipidique dans les interactions lipides-lipides ou lipides-protéines membranaires. Elles pourraient être secondaires à un changement dans la composition lipidique et/ou dans l'interaction des lipides avec les cations bivalents comme le calcium qui sont associés aux têtes polaires des phospholipides et qui contribuent à la stabilisation de la membrane et modulent donc sa fluidité [19]. Par exemple, le lindane, un pesticide organochloré, induit sur les spermatozoïdes humains une augmentation de la fluidité membranaire ainsi qu'une modification du potentiel de membrane et de la concentration intracellulaire de calcium libre [14]. L'incubation d'érythrocytes humains avec l'heptachlore, un pesticide organochloré entraîne une forte diminution de l'anisotropie qui serait due à l'incorporation du pesticide dans la bicouche lipidique. Des changements ultrastructuraux de la membrane plasmique perturberait le transport actif des ions [15]. Dans nos conditions expérimentales, l'augmentation de la fluidité membranaire pourrait être l'un des premiers effets toxiques des deux pesticides testés provoquant ensuite une série d'événements (modification des flux d'ions) susceptibles d'altérer le fonctionnement du spermatozoïde.

L'induction des phénomènes peroxydatifs pourrait être aussi responsable de la toxicité des pesticides sur le spermatozoïde humain [4]. Banerjee et coll. (1999) [5] ont montré sur des leucocytes circulants humains que des organochlorés, (le lindane), des organophosphorés (le malathion) et des carbamates (le propoxur) induisent une augmentation de la peroxydation lipidique couplée à une diminution des protections enzymatiques anti-radicalaires.

La présence de dichloroaniline induit rapidement l'expression de HSP 70, protéine de stress sur une lignée cellulaire Hela, cette induction serait à relier à la cytotoxicité et au caractère lipophile de cette molécule [2]. Ce résultat associé à la présence de HSP 70 dans le spermatozoïde humain [13] pourrait aussi expliquer l'effet toxique de la 3,4-DCA, qui induirait alors certaines protéines de stress.

En raison du caractère lipophile du Diuron et de ses produits de transformation, les membranes biologiques très riches en lipides pourraient être une cible particulière de l'action de ces composés. Cette étude préliminaire suggère que les spermatozoïdes humains puissent être utilisés comme indicateur pour évaluer la toxicité de certains pesticides. Des travaux complémentaires seront nécessaires afin de confirmer ces résultats et de préciser l'importance des produits de photo- ou bio-transformation dans la toxicité du Diuron et le niveau d'action de ces produits. Une atteinte éventuelle de l'activité fonctionnelle du spermatozoïde pourrait aussi être envisagée in vivo. 


\section{RÉFÉRENCES}

1. AGRAWAL R.C., KUMAR S., MEHROTRA N.K. : Micronucleus induction by diuron in mouse bone marrow. Toxicol. Lett., 1996, $89: 1-4$.

2. AIT-AISSA S., PORCHER J., ARRIGO A., LAMBRE C. : Activation of the hsp70 promotor by environmental inorganic and organic chemicals : relationships with cytotoxicity and lipophilicity. Toxicology, 2000, 145: 147-157.

3. AITKEN R.J. : Free radicals, lipid peroxidation and sperm function. Reprod. Fertil. Dev., 1995, 7 : 659668.

4. ALMEIDA M.G., FANINI F., DAVINO S.C, AZNAR A.E., KOCH O.R., BARROS S.B.M. : Pro- and antioxydant parameters in rat liver after short-term exposure to hexachlorobenzene. Hum. Exp. Toxicol., 1997, 16: $257-261$.

5. BANERJEE B.D., SETH V., BHATTACHARYA A., PASHA S.T., CHAKRABORTY A.K. : Biochemical effects of some pesticides on lipid peroxidation and free-radical scavengers. Toxicol. Lett., 1999, 107 : 3347.

6. BARNETT J.B., GANDY J., WILBOURN D., THEUS S.A : Comparison of the immunotoxicity of propanil and its metabolite, 3, 4 dichloroaniline, in C57B1/6 mice. Fundam. Appl. Toxicol., 1992, 18 : 628-631.

7. CONTRERAS H.R., BADILLA J., BUSTOS OBREGON E. : Morphofunctional disturbances of human sperm after incubation with organophosphorate pesticides. Biocell, 1999, 23 :135-141.

8. DE LAMIRANDE E., JIANG H., ZINI A., KODAMA H., GAGNON C. : Reactive oxygen species and sperm physiology. Rev Reprod., 1997, $2: 48-54$

9. GIRAUD M.N., GRIZARD G., BOUCHER D., MOTTA C. : Membrane fluidity predicts the outcome of cryopreservation of human spermatozoa. Hum. Reprod., 2000, $15: 2160-2164$.

10. GRIZARD G., SION B., BAUCHARD, D., BOUCHER D. : Separation and quantification of cholesterol and major phospholipid classes in human semen by highperformance liquid chromatography and light-scattering detection. J. Chromatogr., 2000, 704 : 101-107.

11. HOOGHE R.J., DEVOS S., HOOGHE-PETRES E.L. : Effects of selected herbicides on cytokine production in vitro. Life Sci., 2000, $66: 2519-2525$.

12. KIM JG, PARTHASARATHY S. : Oxidation and the spermatozoa. Semin. Reprod. Endocrinol., 1998, 16 : 235-239.

13. MILLER D., BROUGH S., AL-HARBI O. : Characterization and cellular distribution of human spermatozoal heat shock protein. Hum. Reprod., 1992, 7: 637-645.

14. PALLESCHI S., SILVESTRONI L. : Laurdan fluores- cence spectroscopy reveals a single liquid-crystalline lipid phase and lack of thermotropic phase transitions in the plasma membrane of living human sperm. Biochim. Biophys. Acta., 1996, 1279 : 197-202.

15. SUWALSKY M., BENITES M., VILLENA F., AGUILAR F., SOTOMAYOR C.P. : The organochlorine pesticide heptachlor disrupt the structure of model and cell membrane. Biochim. Biophys. Acta, 1997, 1326: 115-123.

16. TIXIER C., Photo et biotransformation de trois herbicides de type phénylurée : structure, synthèse et écotoxicité des intermédiaires, études sur le terrain. Thèse de l'Université Blaise Pascal, ClermontFerrand, novembre 1999.

17. TOMLIM C. The Pesticide Manual, $10^{\mathrm{eme} e}$ éd. The Bath Press 1994.

18. WANG S.W., CHU C.Y., HSU J.D., WANG C.J. : Haemotoxic effect of phenylurea herbicides in rats: role of haemoglobin-adduct formation splenic toxicity. Food Chem. Toxicol., 1993, 31 : 285-295.

19. YANG Y., HUANG Y.G., TU Y.P. : Divalent cation and lipid-protein interactions of biomembranes. Biosci. Rep. 1993, $13: 143-157$.

\section{ABSTRACT}

Effects of a herbicide Diuron [3-(3,4Dichlorophenyl)-1,1-dimethylurea] and one of its biotransformation products, 3,4-dichloroaniline (3,4-DCA), on human spermatozoa

\section{MALPUECH-BRUGERE, G. GRIZARD, P. BOULE, D. BOUCHER}

Diuron belongs to the family of halogenophenylureas, one of the main groups of herbicides used for more than $\mathbf{4 0}$ years. These herbicides absorb sunlight and can be photochemically transformed in the environment (herbicides are transformed on the soil surface exposed to sunlight) or biotransformed by microorganisms present in soil or in water. The metabolites (chlorohydroxyphenylurea, chlorophenylaniline, respectively) are more toxic than the parent compound, as demonstrated by a bioluminescence inhibition assay performed with a marine bacterium (Vibrio fischeri toxicity test). The lipophilicity of these pesticides makes the cell membrane a target for their action, especially the spermatozoa cell membrane. The aim of this study is to use human 
spermatozoa to evaluate the effect of this urea pesticide and its biotransformed product on the spermatozoa membrane.

We investigated the structural and functional effects of these environmental pollutants on spermatozoa. Three million spermatozoa purified on a 95 / 47.5\% Percoll gradient were suspended in $250 \mu 1$ of modified Earle's medium (without phenol red) supplemented with $7.5 \%$ of human decomplemented serum. Pesticides (Diuron or 3,4-dichloroaniline (3,4DCA)) were added at a final concentration of $0.1 ; 1$ and $5 \mathrm{mM}$. Samples were incubated at room temperature for 24 hours. We show that both Diuron and 3,4-DCA decrease motility and vitality of spermatozoa incubated with the highest concentration of pesticides. Our preliminary results show that the effects are more rapid and more intense with the biotransformed product $(3,4-D C A)$ than with Diuron. Addition of herbicide to human spermatozoa increases membrane fluidity, assessed by measuring the fluorescence polarisation anisotropy with a fluorescent probe: 1,6diphenyl-1,3,5-hexatriene (DPH). Changes in membrane fluidity may be a primary toxic effect of these herbicides.

These results suggest that human spermatozoa may constitute a valuable indicator of the toxic effects of pesticides.

Key words: spermatozoa, membrane, fluidity, pesticide, herbicide 